This item was submitted to Loughborough's Research Repository by the author.

Items in Figshare are protected by copyright, with all rights reserved, unless otherwise indicated.

\title{
Outage-constrained robust power allocation for downlink MC-NOMA with imperfect SIC
}

PLEASE CITE THE PUBLISHED VERSION

http://doi.org/10.1109/ICC.2018.8422364

PUBLISHER

(C) IEEE

VERSION

AM (Accepted Manuscript)

LICENCE

CC BY-NC-ND 4.0

\section{REPOSITORY RECORD}

Li, Shenhong, Mahsa Derakhshani, and Sangarapillai Lambotharan. 2019. "Outage-constrained Robust Power Allocation for Downlink MC-NOMA with Imperfect SIC”. figshare. https://hdl.handle.net/2134/28466. 


\title{
Outage-Constrained Robust Power Allocation for Downlink MC-NOMA with Imperfect SIC
}

\author{
Shenhong Li, Mahsa Derakhshani, Sangarapillai Lambotharan \\ Wolfson School of Mechanical, Electrical and Manufacturing Engineering, Loughborough University, UK \\ Email: S.Li3@lboro.ac.uk; M.Derakhshani@lboro.ac.uk; S.Lambotharan@lboro.ac.uk
}

\begin{abstract}
In this paper, we study power allocation for downlink multi-carrier non-orthogonal multiple access (MC-NOMA) systems and examine the effects of residual cancellation errors resulting from imperfect successive interference cancellation (SIC) on the system performance. In the presence of random SIC errors, we study outage probability of minimum reserved rate for individual user and formulate outage-constrained robust optimization to minimize the total transmit power. Since the problem is non-convex due to probabilistic constraints, complementary geometric programming (CGP) and arithmetic geometric mean approximation (AGMA) technique are employed to transform it into a convex form. An efficient iterative algorithm with low computational complexity is developed to solve the optimization problem. Simulation results demonstrate the performance of robust MC-NOMA with imperfect SIC and compare that to non-robust MC-NOMA and orthogonal multiple access (OMA) schemes.
\end{abstract}

Index Terms-MC-NOMA, 5G, robust optimization, imperfect SIC, outage probability, complementary geometric programming.

\section{INTRODUCTION}

Enhancing the capacity of wireless communication networks is essential for meeting the demand for continuous expansion of the network and services. Multi-carrier nonorthogonal multiple access (MC-NOMA) is one of the promising techniques proposed aiming to meet the targets set for the fifth generation of wireless networks $(5 \mathrm{G})$ and beyond [1]. In contrast to orthogonal multiple access (OMA) such as orthogonal frequency-division multiple access (OFDMA), NOMA can serve multiple users in the same frequency subcarrier by utilizing the power domain multiplexing and successive interference cancellation (SIC) [2]. Thus, NOMA can effectively enhance spectrum efficiency compared to OFDMA scheme [3].

In downlink NOMA, the SIC process is utilized at the receiver side to decode the superposition of various user signals from a base station (BS). The user with high power level is decoded first while the other user signals are treated as interference. After this decoding stage, the high power signal is removed from the composition signal, and the iterative process is repeated until the last user signal is decoded [4], [5].

There have been many recent works on NOMA [6] studying user pairing/clustering, hybrid multiple access, multiple-

This work has been supported by the Engineering and Physical Science Research Council of the UK, EPSRC, under the grant EP/M015475. input-multiple-output (MIMO)-NOMA, and cross-layer optimization. Particularly, the works in [7] and [8] studied the effects of user pairing and user clustering on the performance of NOMA respectively. The power allocation problem for downlink OFDMA combined with NOMA system was solved by applying difference of convex functions (DC) programming algorithm in [9]. A MIMO-NOMA system for downlink and uplink transmissions was investigated by applying the concept of signal alignment [10]. The application of downlink MCNOMA to support isolation among different slices in virtualized wireless networks is investigated in [11].

Most of existing works on NOMA are based on the idealistic assumption of perfect subtraction of previous user signals in SIC with no residual interference incurred. However, SIC error propagation can result from various sources such as synchronization, channel estimation errors and imperfect decoding [12], and thus, can jeopardize the NOMA benefits in practice [13]. Therefore, it would be of interest to study how imperfect SIC could affect the NOMA performance. In [12], SIC error propagation is studied by modeling it as a deterministic parameter for resource allocation in a downlink MIMO-NOMA aiming to maximize throughput. In [14], the bit error rate performance of a two-user downlink NOMA system is investigated under perfect and imperfect SIC conditions respectively. However, further research is required on outageconstrained resource allocation in NOMA systems to achieve robustness against random SIC errors.

In this work, we consider error propagation in the downlink MC-NOMA system due to imperfect SIC and investigate a probabilistic robust power allocation design to handle uncertain parameters in the system. Assuming normally distributed errors, we first study the outage probability of minimum reserved data rate for each individual user employing the Chebyshev-Cantelli inequality. Then, we formulate a robust optimization problem aiming to minimize the total transmit power subject to outage probability constraints for qualityof-service $(\mathrm{QoS})$ provisioning for individual users. Exploiting the proposed approximation on outage probabilities, the robust optimization is converted into a deterministic form, which is a non-convex optimization problem.

To solve the resulting non-convex and NP-hard optimization, first, the problem is cast into CGP by applying several transformations [15]. To reach the optimal solution of the CGP, an iterative algorithm is then developed by applying AGMA 
approximations and solving a series of GP problems based on the successive convex approximation (SCA) [16], [17].

Via simulation results, we evaluate the effectiveness of proposed outage-constrained robust algorithm to improve the power efficiency in comparison with non-robust MC-NOMA and OFDMA techniques under different settings such as the number of users and minimum reserved rate. The results confirm that the proposed robust technique could preserve the superiority of MC-NOMA to OFDMA even in the presence of SIC errors, while non-robust MC-NOMA shows very close performance to OFDMA.

The remainder of this paper is organized as follows. Section II presents the system model. In Section III, the robust optimization problem is formulated subject to outage constraints. In Section IV, we study the outage probability constraint for the minimum reserved rate of individual users. The iterative algorithm and the techniques to convert the non-convex problem into convex form are introduced in Section V. Simulation results and conclusions are presented in Sections VI and VII, respectively.

\section{SySTEM MODEL}

We consider a MC-NOMA downlink system with a single base station (BS) that serves a set of users denoted by $\mathcal{K}=\{1, \cdots K\}$. Users are uniformly distributed within a circle where the base station is located at the center of this circle. The available bandwidth denoted by $B$ is divided into a set of sub-carriers $\mathcal{N}=\{1, \cdots N\}$. The channel gain between the BS and the user $i$ at sub-carrier $n$ (denoted by $h_{i, n}$ ), that reflects large-scale and small-scale fading, is given by

$$
h_{i, n}=\chi_{i, n} d_{i}^{-\lambda}
$$

where $\chi_{i, n}$ denotes the channel coefficient of small scale fading; $d_{i}$ denotes the distance between user $i$ and the BS; and $\lambda$ denotes the path loss coefficient.

In each sub-carrier $n$, all users are sorted based on their channel gains in a decreasing order $\left|h_{1, n}\right|>\left|h_{2, n}\right|>\cdots>$ $\left|h_{K, n}\right|$. The receiver of user ranked $i$ aims to cancel the interference from any other users whose indices are greater than $i$ using SIC. However, the remaining user signals, whose indices are lower than $i$, are treated as unresolvable interference. Thus, the received signal-to-interference-plus-noise ratio (SINR) of user $i$ at the sub-carrier $n$ is given by

$$
\operatorname{SINR}_{i, n}^{\mathrm{NOMA}}=\frac{\beta_{i, n} h_{i, n}}{\sigma_{i, n}^{2}+h_{i, n} \sum_{j=1}^{i-1} \beta_{j, n}+I_{i, n}^{e}}
$$

where $\beta_{i, n}$ is the power allocation coefficient for the $i^{\text {th }}$ user at sub-carrier $n$ and $\sigma_{i, n}^{2}$ is the additive white Gaussian noise (AWGN) power. Moreover, $I_{i, n}^{e}$ is the interference resulting from imperfect cancellation of the transmissions of users $i<$ $j \leq K$ during SIC, which will be discussed in more details in the next section.
Consequently, the data rate of user $i$ at the sub-carrier $n$ can be written as

$$
\begin{aligned}
& R_{i, n}^{\mathrm{NOMA}}=R_{i, n}=\log \left(1+\mathrm{SINR}_{i, n}^{\mathrm{NOMA}}\right) \\
& \quad=\log \left(1+\frac{\beta_{i, n} h_{i, n}}{\sigma_{i, n}^{2}+h_{i, n} \sum_{j=1}^{i-1} \beta_{j, n}+I_{i, n}^{e}}\right)
\end{aligned}
$$

where the achieved rate is given in [nats/s/Hz].

For QoS support purposes, we further assume that each user $i \in \mathcal{K}$ requires a minimum reserved rate of $R_{i}^{\text {rsv }}$, which can be expressed as

$$
R_{i}=\sum_{n \in \mathcal{N}} R_{i, n}^{\mathrm{NOMA}} \geq R_{i}^{\mathrm{rsv}}, \forall i \in \mathcal{K}
$$

where $R_{i}$ represents the data rate of user $i$.

\section{Robust Optimization PROBlem}

In this section, considering that SIC is not perfect and hence random residual cancellation errors occur, we formulate a robust optimization problem to minimize the transmit power, while limiting the outage probability of minimum reserved rate for each user. In presence of random SIC errors, $I_{i, n}^{e}$ can be presented as

$$
I_{i, n}^{e}=h_{i, n} \sum_{j=i+1}^{K} \beta_{j, n}\left\|e_{j, n}\right\|^{2}
$$

which represents the unresolvable interference due to imperfect cancellation of the transmission of users $i+1<j \leq K$ during SIC, where $e_{j, n}=x_{j, n}-\hat{x}_{j, n}$ is the difference between the estimated (i.e., $\hat{x}_{j, n}$ ) and actual signal (i.e., $x_{j, n}$ ) transmitted for user $j$ at sub-carrier $n$. In case of perfect SIC, these two signals are equal and the value of $e_{j, n}$ will be zero. Otherwise, some portion of the received power at user $i, h_{i, n} \beta_{j, n}$, remains as interference due to SIC errors.

We model the SIC errors as $e_{j, n} \sim \mathcal{C N}\left(0, \sigma_{e}^{2}\right)$ and as a result, $\frac{1}{\sigma^{2}}\left\|e_{j, n}\right\|^{2}$ is a random variable, which has a chisquared distribution with two degrees of freedom. Such model is assumed considering the potential sources of error such as thermal noise and asynchronization which follow Gaussian distribution as shown in [18].

Due to randomness of SIC errors, we introduce outage probability constraints for different users, limiting the probability that data rate of each user drops below the minimum reserved rate. Thus, the outage-probability constraints are written as

$$
\operatorname{Pr}\left[R_{i} \leq R_{i}^{\mathrm{rsv}}\right] \leq \epsilon, \forall i \in \mathcal{K}
$$

where $0 \leq \epsilon \leq 1$ denotes the maximum tolerable outage probability for each user. In other words, $\operatorname{Pr}\left[R_{i} \geq R_{i}^{\text {rsv }}\right]$ is restricted to be larger than a certain threshold for each user.

$$
\text { C1 }: \operatorname{Pr}\left[R_{i} \geq R_{i}^{\text {rsv }}\right] \geq 1-\epsilon, \forall i \in \mathcal{K} .
$$

Considering $\mathrm{C} 1$ constraints, we aim to solve the following robust optimization problem to minimize the total transmit power.

$$
\min _{\boldsymbol{\beta}} \sum_{n \in \mathcal{N}} \sum_{i \in \mathcal{K}} \beta_{i, n},
$$




\section{OUtage Probability Analysis}

In order to solve the robust optimization problem (8), here, we analytically study the outage probability of minimum reserved rate for each user.

According to Chebyshev-Cantelli inequality, we have

$$
\operatorname{Pr}[X-\mathbf{E}[X] \geq \eta] \leq \frac{\operatorname{Var}[X]}{\operatorname{Var}[\mathbf{X}]+\eta^{2}}
$$

for any random variable $X$ where $\eta>0$.

Using this bound, the outage probability constraint (i.e., C1) can be relaxed into deterministic form as below

$$
\frac{\operatorname{Var}\left[R_{i}\right]}{\operatorname{Var}\left[R_{i}\right]+\left(R_{i}^{\text {rv }}-\mathbf{E}\left[R_{i}\right]\right)^{2}} \geq 1-\epsilon
$$

After some mathematical manipulations, we can express the outage probability constraint as

$$
\mathbf{E}\left[R_{i}\right]+\sqrt{\operatorname{Var}\left[R_{i}\right] \frac{\epsilon}{1-\epsilon}} \geq R_{i}^{\mathrm{rsv}} .
$$

Thus, we need to calculate the mean and variance of $R_{i}$.

For the sake of simplicity, let define

$$
R_{i}=\sum_{n \in \mathcal{N}} \log \left(\gamma_{i, n}\right)
$$

where

$$
\begin{aligned}
\gamma_{i, n} & =1+\operatorname{SINR}_{i, n}^{\mathrm{NOMA}}=1+\frac{a_{i, n}}{b_{i, n}+I_{i, n}^{e}}, \\
a_{i, n} & =\beta_{i, n} h_{i, n}, \\
b_{i, n} & =\sigma_{i, n}^{2}+h_{i, n} \sum_{j=1}^{i-1} \beta_{j, n} .
\end{aligned}
$$

Based on (12), the mean and variance of $R_{i}$ can be written as

$$
\begin{aligned}
& \mathbf{E}\left[R_{i}\right]=\sum_{n \in \mathcal{N}} \mathbf{E}\left[\log \left(\gamma_{i, n}\right)\right] \\
& \operatorname{Var}\left[R_{i}\right]=\sum_{n \in \mathcal{N}} \operatorname{Var}\left[\log \left(\gamma_{i, n}\right)\right]
\end{aligned}
$$

By applying the Taylor-series expansion of logarithmic function, we can approximate the rate function and then calculate $\mathbf{E}\left[\log \left(\gamma_{i, n}\right)\right]$ and $\operatorname{Var}\left[\log \left(\gamma_{i, n}\right)\right]$. A truncated Taylor series of logarithmic function with two terms can be represented as

$$
\log \left(\gamma_{i, n}\right) \approx \log \left(\mathbf{E}\left(\gamma_{i, n}\right)\right)+\frac{\gamma_{i, n}-\mathbf{E}\left[\gamma_{i, n}\right]}{\mathbf{E}\left[\gamma_{i, n}\right]}
$$

Taking the expected value and variance of the both sides in (18), we obtain

$$
\begin{aligned}
& \mathbf{E}\left[\log \left(\gamma_{i, n)}\right] \approx \log \left(\mathbf{E}\left[\gamma_{i, n}\right]\right)\right. \\
& \operatorname{Var}\left[\log \left(\gamma_{i, n}\right)\right] \approx \frac{\operatorname{Var}\left[\gamma_{i, n}\right]}{\mathbf{E}^{2}\left[\gamma_{i, n}\right]}
\end{aligned}
$$

Approximating $\gamma_{i, n}\left(I_{i, n}^{e}\right)=1+\frac{a_{i, n}}{b_{i, n}+I_{i, n}^{e}}$ using Taylor-series expansion with two terms, we can calculate $\mathbf{E}\left[\gamma_{i, n}\right]$ and $\operatorname{Var}\left[\gamma_{i, n}\right]$.

$$
\begin{aligned}
& \gamma_{i, n}\left(I_{i, n}^{e}\right) \approx 1+\frac{a_{i, n}}{b_{i, n}+\mathbf{E}\left[I_{i, n}^{e}\right]} \\
& \left.-\frac{a_{i, n}}{\left(b_{i, n}+\mathbf{E}\left[I_{i, n}^{e}\right]\right)^{2}}\left(I_{i, n}^{e}\right]-\mathbf{E}\left[I_{i, n}^{e}\right]\right)
\end{aligned}
$$

Consequently, we have

$$
\begin{aligned}
& \mathbf{E}\left[\gamma_{i, n}\right] \approx 1+\frac{a_{i, n}}{b_{i, n}+\mathbf{E}\left[I_{i, n}^{e}\right]} \\
& \operatorname{Var}\left[\gamma_{i, n}\right] \approx \frac{a_{i, n}^{2}}{\left(b_{i, n}+\mathbf{E}\left[I_{i, n}^{e}\right]\right)^{4}} \operatorname{Var}\left[I_{i, n}^{e}\right]
\end{aligned}
$$

Therefore, considering (16), (17), (19), (20), (22) and (23), we have

$$
\begin{aligned}
& \mathbf{E}\left[R_{i}\right] \approx \sum_{n \in \mathcal{N}} \log \left(1+\frac{a_{i, n}}{b_{i, n}+\mathbf{E}\left[I_{i, n}^{e}\right]}\right) \\
& \operatorname{Var}\left[R_{i}\right] \approx \sum_{n \in \mathcal{N}}\left(\frac{a_{i, n}}{b_{i, n}+\mathbf{E}\left[I_{i, n}^{e}\right]}\right)^{2} \frac{\operatorname{Var}\left[I_{i, n}^{e}\right]}{\left(a_{i, n}+b_{i, n}+\mathbf{E}\left[I_{i, n}^{e}\right]\right)^{2}}
\end{aligned}
$$

Finally, replacing (24) and (25) in (11), the outage constraint can be represented in a deterministic form as

$$
\begin{aligned}
& \hat{\mathbf{C}} 1: \sum_{n \in \mathcal{N}} \log \left(1+\frac{a_{i, n}}{b_{i, n}+\mathbf{E}\left[I_{i, n}^{e}\right]}\right)+\sqrt{\frac{\epsilon}{1-\epsilon}} \times \\
& \sqrt{\sum_{n \in \mathcal{N}}\left(\frac{a_{i, n}}{b_{i, n}+\mathbf{E}\left[I_{i, n}^{e}\right]}\right)^{2} \frac{\operatorname{Var}\left[I_{i, n}^{e}\right]}{\left(a_{i, n}+b_{i, n}+\mathbf{E}\left[I_{i, n}^{e}\right]\right)^{2}}} \geq R_{i}^{\mathrm{rvv}}, \forall i
\end{aligned}
$$

where

$$
\begin{aligned}
& \mathbf{E}\left[I_{i, n}^{e}\right]=2 h_{i, n} \sum_{j=i+1}^{K} \beta_{j, n} \sigma_{e}^{2} \\
& \operatorname{Var}\left[I_{i, n}^{e}\right]=4 h_{i, n}^{2} \sum_{j=i+1}^{K} \beta_{j, n}^{2} \sigma_{e}^{4}
\end{aligned}
$$

To deal with the outage constraint $\hat{\mathbf{C}} 1$, we define two new variables $X_{i, n}$ and $Y_{i}$ as

$$
\begin{aligned}
& X_{i, n}=\log \left(1+\frac{a_{i, n}}{b_{i, n}+\mathbf{E}\left[I_{i, n}^{e}\right]}\right) \\
& Y_{i}=\sqrt{\sum_{n \in \mathcal{N}}\left(\frac{a_{i, n}}{b_{i, n}+\mathbf{E}\left[I_{i, n}^{e}\right]}\right)^{2} \frac{\operatorname{Var}\left[I_{i, n}^{e}\right]}{\left(a_{i, n}+b_{i, n}+\mathbf{E}\left[I_{i, n}^{e}\right]\right)^{2}}}
\end{aligned}
$$

Considering new variables, we transform $\hat{\mathbf{C}} 1$ into three constraints as

$$
\begin{aligned}
& \text { C2 }: \sum_{n \in \mathcal{N}} X_{i, n}+\sqrt{\frac{\epsilon}{1-\epsilon}} Y_{i} \geq R_{i}^{\mathrm{rsv}}, \forall i \in \mathcal{K}, \forall n \in \mathcal{N} \\
& \text { C3 }: X_{i, n} \leq \log \left(1+\frac{a_{i, n}}{b_{i, n}+2 h_{i, n} \sum_{j=i+1}^{K} \beta_{j, n} \sigma_{e}^{2}}\right) \\
& \forall i \in \mathcal{K}, \forall n \in \mathcal{N} \\
& \text { C4 }: Y_{i}^{2} \leq \sum_{n \in \mathcal{N}}\left(\frac{a_{i, n}}{b_{i, n}+2 h_{i, n} \sum_{j=i+1}^{K} \beta_{j, n} \sigma_{e}^{2}}\right)^{2} \times \\
& \quad \frac{4 h_{i, n}^{2} \sum_{j=i+1}^{K} \beta_{j, n}^{2} \sigma^{4}}{\left(a_{i, n}+b_{i, n}+2 h_{i, n} \sum_{j=i+1}^{K} \beta_{j, n} \sigma_{e}^{2}\right)^{2}}, \forall i \in \mathcal{K}, \forall n \in \mathcal{N}
\end{aligned}
$$


Eventually, the robust optimization problem (8) can be expressed as

$$
\begin{aligned}
& \min _{\boldsymbol{\beta}, \boldsymbol{X}, \boldsymbol{Y}} \sum_{n \in \mathcal{N}} \sum_{i \in \mathcal{K}} \beta_{i, n}, \\
& \text { subject to : } \mathbf{C} \mathbf{2}-\mathbf{C 4} .
\end{aligned}
$$

where $\boldsymbol{\beta}=\left[\beta_{i, n}\right]_{K \times N}, \boldsymbol{X}=\left[X_{i, n}\right]_{K \times N}$, and $\boldsymbol{Y}=\left[Y_{i}\right]_{1 \times K}$.

\section{PROPOSED ALGORITHM}

The optimization problem in (34) is non-convex and inherently complex to find its optimal solution. In order to solve this optimization problem, we deploy an iterative method of successive convex approximations, in which the non-convex function is transformed into convex one in each iteration. In this regard, we first cast the problem into CGP via several transformations techniques. We refer the interested readers to [15], [16], [19] for further reading on CGP background. Then, applying AGMA approximations, the optimal solution of (34) can be achieved by solving a series of the geometric programming (GP) problems.

According to CGP definition, the inequality constraints need to be in the form of a ratio between two posynomials. Here, to convert constraints in a form acceptable by a CGP standard, we first reformulate $\mathbf{C} 2$ as

$$
\hat{\mathbf{C}} 2: \frac{R_{i}^{\mathrm{rsv}}}{\sum_{n \in \mathcal{N}} X_{i, n}+\sqrt{\frac{\epsilon}{1-\epsilon}} Y_{i}} \leq 1
$$

For C3, we transform it into

$$
e^{X_{i, n}} \leq\left(1+\frac{a_{i, n}}{b_{i, n}+2 h_{i, n} \sum_{j=i+1}^{K} \beta_{j, n} \sigma_{e}^{2}}\right)
$$

Since $e^{X_{i, n}}$ is not acceptable in a CGP form, we use the truncated Taylor series to approximate it and then (36) can be transformed to

$$
\hat{\mathbf{C}} 3: \sum_{m=0}^{M} \frac{X_{i, n}^{m}}{m !} \frac{\left(b_{i, n}+2 h_{i, n} \sum_{j=i+1}^{K} \beta_{j, n} \sigma_{e}^{2}\right)}{\left(a_{i, n}+b_{i, n}+2 h_{i, n} \sum_{j=i+1}^{K} \beta_{j, n} \sigma_{e}^{2}\right)} \leq 1
$$

Finally, in order to deal with $\mathbf{C 4}$, it is divided into two different constraints by introducing a new variable $W_{i, n}$ as

$$
\begin{aligned}
& \hat{\mathbf{C}} 4-1: \frac{Y_{i}^{2}}{\sum_{n \in \mathcal{N}} W_{i, n}} \leq 1 \\
& \hat{\mathbf{C}} 4-2:\left(\frac{W_{i, n}\left(b_{i, n}+2 h_{i, n} \sum_{j=i+1}^{K} \beta_{j, n} \sigma_{e}^{2}\right)^{2}}{4 a_{i, n}^{2} h_{i, n}^{2} \sum_{j=i+1}^{K} \beta_{j, n}^{2} \sigma_{e}^{4}}\right) \times \\
& \left(a_{i, n}+b_{i, n}+2 h_{i, n} \sum_{j=i+1}^{K} \beta_{j, n} \sigma_{e}^{2}\right)^{2} \leq 1
\end{aligned}
$$

Considering the transformed forms of constraints, we have

$$
\min _{\boldsymbol{\beta}, \boldsymbol{X}, \boldsymbol{Y}, \boldsymbol{W}} \sum_{i=1}^{K} \sum_{n=1}^{N} \beta_{i, n}(t)
$$

which is a CGP problem. To solve the CGP problem, consider $t$ as the iteration number. In each iteration $t$, the posynomial functions in the denominators of different constraints are approximated as monomial functions applying ArithmeticGeometric Mean Approximation (AGMA). In doing so, a CGP problem is converted to a GP, which can be effectively solved by available software packages, e.g., CVX, in each iteration.

At iteration $t, \hat{\mathbf{C}} 2$ can be approximated as

$$
\tilde{\mathbf{C}} 2: R_{i}^{\mathrm{rsv}} \prod_{n \in \mathcal{N}}\left(\frac{X_{i, n}(t)}{\alpha_{i, n}(t)}\right)^{-\alpha_{i, n}(t)}\left(\frac{\sqrt{\frac{\epsilon}{1-\epsilon}} Y_{i}(t)}{\omega_{i}(t)}\right)^{-\omega_{i}(t)} \leq 1
$$

where for $\forall i \in \mathcal{K}$ and $\forall n \in \mathcal{N}$, we have

$$
\begin{aligned}
& \alpha_{i, n}(t)=\frac{X_{i, n}(t-1)}{\sum_{n \in \mathcal{N}} X_{i, n}(t-1)+\sqrt{\frac{\epsilon}{1-\epsilon}} Y_{i}(t-1)} \\
& \omega_{i}(t)=\frac{\sqrt{\frac{\epsilon}{1-\epsilon}} Y_{i}(t-1)}{\sum_{n \in \mathcal{N}} X_{i, n}(t-1)+\sqrt{\frac{\epsilon}{1-\epsilon}} Y_{i}(t-1)}
\end{aligned}
$$

Similarly, $\mathbf{C} 3$ can be approximated by applying AGMA method as

$$
\begin{aligned}
& \tilde{\mathbf{C}} 3: \sum_{m=0}^{M} \frac{X_{i, n}(t)^{m}}{m !}\left(\sigma_{i, n}^{2}+h_{i, n} \sum_{j=1}^{i-1} \beta_{j, n}(t)\right. \\
& \left.+2 h_{i, n} \sum_{j=i+1}^{K} \beta_{j, n}(t) \sigma_{e}^{2}\right)\left(\frac{\sigma_{i, n}^{2}}{s_{i, n}(t)}\right)^{-s_{i, n}(t)} \times \\
& \left(\frac{\beta_{i, n}(t) h_{i, n}}{r_{i, n}(t)}\right)^{-r_{i, n}(t)} \prod_{j=1}^{i-1}\left(\frac{h_{i, n} \beta_{j, n}(t)}{g_{i, j, n}(t)}\right)^{-g_{i, j, n}(t)} \times \\
& \prod_{j=i+1}^{K}\left(\frac{2 h_{i, n} \beta_{j, n}(t) \sigma_{e}^{2}}{f_{i, j, n}(t)}\right)^{-f_{i, j, n}(t)} \leq 1
\end{aligned}
$$

where

$$
\begin{aligned}
& s_{i, n}(t)=\frac{\sigma_{i, n}^{2}}{z_{i, n}(t)} \\
& g_{i, j, n}(t)=\frac{\beta_{j, n}(t-1) h_{i, n}}{z_{i, n}(t)} \\
& f_{i, j, n}(t)=\frac{2 \beta_{j, n}(t-1) h_{i, n} \sigma_{e}^{2}}{z_{i, n}(t)} \\
& r_{i, n}(t)=\frac{\beta_{i, n}(t-1) h_{i, n}}{z_{i, n}(t)} \\
& z_{i, n}(t)=\sigma_{i, n}^{2}+\beta_{i, n}(t-1) h_{i, n}+h_{i, n} \sum_{j=1}^{i-1} \beta_{j, n}(t-1) \\
& +2 h_{i, n} \sum_{j=i+1}^{K} \beta_{j, n}(t-1) \sigma_{e}^{2}
\end{aligned}
$$


$\overline{\text { Algorithm } 1 \text { : Iterative Algorithm } 1 \text { Based on CGP for NOMA }}$

Initialization: Set $t=1, \boldsymbol{\beta}(t-1)=[\mathbf{1}]$ and $\boldsymbol{X}(t-1)=[\mathbf{1}]$, where $\mathbf{1}$ is a matrix, $\boldsymbol{Y}(t-1)=[\mathbf{1}]$ and $\boldsymbol{W}=[\mathbf{1}]$,where there are vectors $C^{1 \times K}$. Repeat:

Step 1: Update $\alpha_{i}(t), \omega_{i}(t)$ from (42) and (43),

Step 2: Update $s_{i, n}(t), g_{i, j, n}(t), f_{i, j, n}(t), r_{i, n}(t)$, and $z_{i, n}(t)$ from (45)-(49),

Step 3: Update $\theta_{i, n}(t)$ and $\delta_{j, n}(t)$ from (52) and (53),

Step 4: Find optimal value of $\boldsymbol{\beta}^{*}(t), \boldsymbol{X}^{*}(t), \boldsymbol{Y}^{*}(t), \boldsymbol{W}^{*}(t)$ from (54) via CVX [20]. We also need to update the value of $\boldsymbol{\beta}(t-1), \boldsymbol{X}(t-1), \boldsymbol{Y}(t-1)$ and $\boldsymbol{W}(t-1)$

Until: $\left\|\boldsymbol{\beta}^{*}(t)-\boldsymbol{\beta}^{*}(t-1)\right\| \leq \varepsilon$.

Applying AGMA, at iteration $t, \hat{\mathbf{C}} 4-\mathbf{1}$ and $\hat{\mathbf{C}} 4-2$ can be expressed as

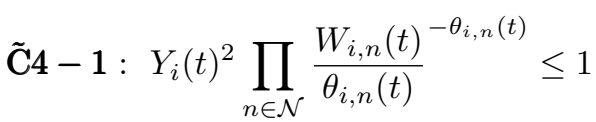

$$
\begin{aligned}
& \tilde{\mathbf{C}} 4-\mathbf{2}:\left(\sigma_{i, n}^{2}+h_{i, n} \sum_{j=1}^{i-1} \beta_{j, n}(t)+2 h_{i, n} \sum_{j=i+1}^{K} \beta_{j, n}(t) \sigma_{e}^{2}\right) \times \\
& \left(\beta_{i, n}(t) h_{i, n}+\sigma_{i, n}^{2}+h_{i, n} \sum_{j=1}^{i-1} \beta_{j, n}(t)+2 h_{i, n} \sum_{j=i+1}^{K} \beta_{j, n}(t) \sigma_{e}^{2}\right) \\
& \times\left(\frac{W_{i, n}(t)}{4 \beta_{i, n}(t)^{2} h_{i, n}^{4} \sigma_{e}^{4}}\right) \prod_{j=i+1}^{K}\left(\frac{\beta_{j, n}^{2}(t)}{\delta_{j, n}(t)}\right)^{-\delta_{j, n}(t)} \leq 1
\end{aligned}
$$

where

$$
\begin{aligned}
& \theta_{i, n}(t)=\frac{W_{i, n}(t-1)}{\sum_{n \in \mathcal{N}} W_{i, n}(t-1)} \\
& \delta_{j, n}(t)=\frac{\beta_{j, n}^{2}(t-1)}{\sum_{j=i+1}^{K} \beta_{j, n}^{2}(t-1)}
\end{aligned}
$$

Then, at each iteration $t$, we solve the following GP problem

$$
\min _{\boldsymbol{\beta}(t), \boldsymbol{X}(t), \boldsymbol{Y}(t), \boldsymbol{W}(t)} \sum_{i=1}^{K} \sum_{n=1}^{N} \beta_{i, n}(t)
$$

subject to: $\tilde{\mathrm{C}} 2, \tilde{\mathrm{C}} 3, \tilde{\mathrm{C}} 4-1 \& \tilde{\mathrm{C}} 4-2$

Different steps of the proposed iterative algorithm that need to be performed until convergence are presented in Algorithm 1.

\section{Simulation Results}

In this section, we evaluate the power allocation performance of the proposed algorithm for MC-NOMA, and compare it with the non-robust MC-NOMA and OFDMA schemes. We consider a unit circle with base station located at the center and several users distributed randomly and uniformly within this circle. The channel gains are modeled as $h_{i, n}=\chi_{i, n} d_{i}^{-\lambda}$. We assume Rayleigh fading channel and the small scale fading $\chi_{i, n}$ thus follows an exponential distribution with parameter 1. $d_{i} \geq 0$ denotes the distance between the BS and the $i^{\text {th }}$ user. The path loss exponent is $\lambda=3$. The variance

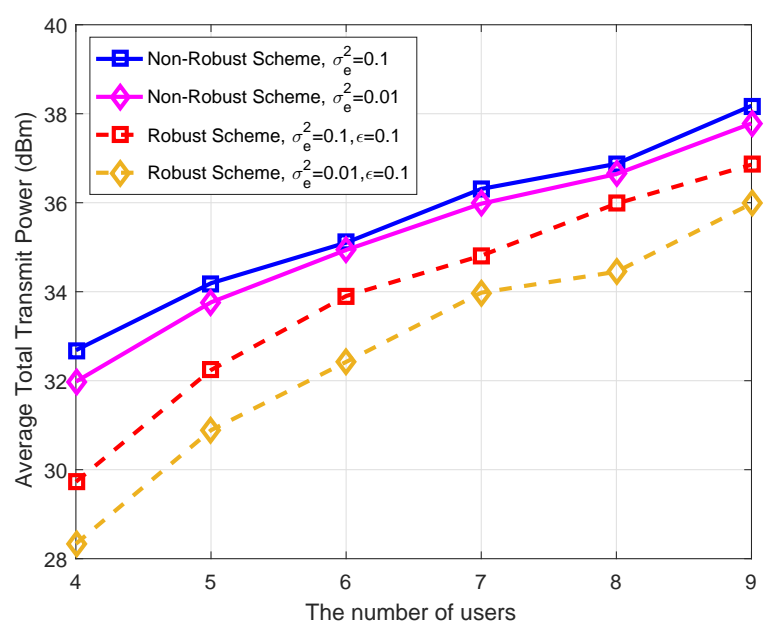

Fig. 1. Average total transmit power versus users

of the additive white Gaussian noise is normalized to one, i.e., $\sigma_{i, n}^{2}=1$, which is assumed to be equal for all users. The variances of SIC error are set to $\sigma_{e}^{2}=0.1$ and 0.01 unless otherwise mentioned. For the sake of clarity, only the maximum and minimum values of SIC error variance are shown in the figures. As expected, the total transmit power increases with the number of users, rate reservations, and the level of SIC errors, while it decreases with increasing outage probability constraint. The relationships of them are depicted in the following figures. The results are taken over the average of 100 Monte-Carlo channel realizations. It should be noted that Algorithm 1 has been implemented in Matlab for the case of outage-constrained robust MC-NOMA. For the case of nonrobust MC-NOMA and OFDMA, the algorithms proposed in [11] have been implemented in Matlab.

Figure 1 depicts the effect of the number of users on the system performance for outage-constrained robust MC-NOMA and non-robust MC-NOMA with imperfect SIC, for $R_{i}^{\text {rsv }}=1$ nats $/ \mathrm{s} / \mathrm{Hz}$ and the fixed number of sub-carriers $N=8$. In the two cases, it can be seen that as the number of users increases, the average total transmit power required also increases. The reason is that the BS needs to transmit at a higher power level to satisfy the minimum data rate requirements for all users. Power level required for high value of SIC error $\sigma_{e}^{2}=0.1$ is always greater than the one for low SIC error, i.e., $\sigma_{e}^{2}=$ 0.01 . Moreover, it is clear that the proposed outage-constrained robust scheme for MC-NOMA provides considerable power saving as compared to the non-robust MC-NOMA.

Figure 2 depicts the relationship between the average total transmit power and minimum reserved rate. We consider a system with 8 sub-carriers which can be shared by 4 users. Obviously, the average transmit power increases with the increase of $R_{i}^{\text {rsv }}$. This happens because it is more difficult to ensure users with poor channel gains to satisfy quality of service (QoS) requirements. We consider not only the aforementioned two cases, but also the different value of 


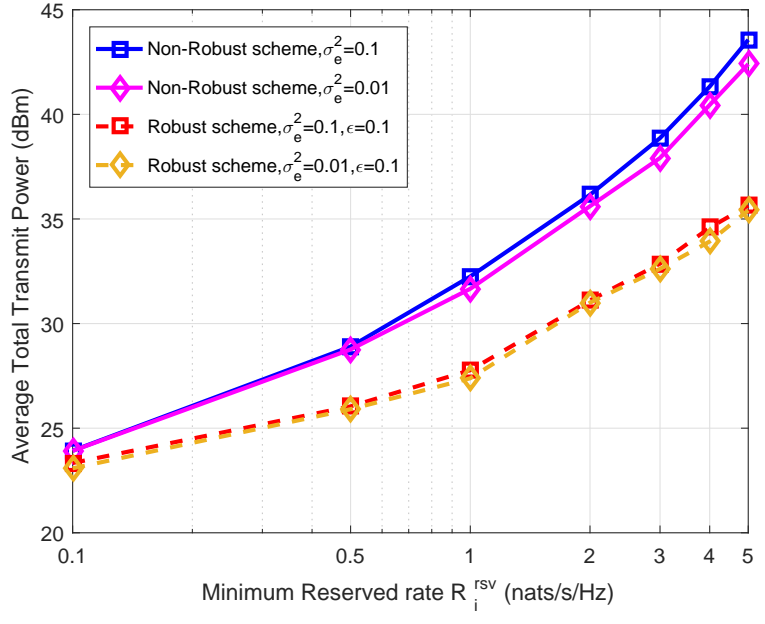

Fig. 2. Average total transmit power versus $R_{i}^{\text {rsv }}$

$\sigma_{e}^{2}=0.01$. It turns out that the large error will require more power in MC-NOMA system. This result also confirms the efficacy of the proposed robust power allocation scheme to save more power. Moreover, evidently, the performance gap is increased as $R_{i}^{\text {rsv }}$ increases. This demonstrates the significant superiority of the proposed robust algorithm in case of stricter QoS requirements.

In Figure 3, we provide simulation results to compare the performance of outage-constrained robust MC-NOMA and the non-robust MC-NOMA in the presence of SIC errors, as well as OFDMA in two different ring-shaped regions. In the first region, users are uniformly located at the cell center where $d_{i} \in[0.1,0.4]$. In the second region, users are distributed at the cell edge with distance from 0.4 to 1 . It can be seen that the total transmit power for the case of cell center is less than that of the cell edge. In addition, MC-NOMA provides best power efficient performance with outage-constrained robust scheme. Another significant observation is that power efficiency is increased with robust scheme MC-NOMA as compared to the OFDMA scheme for the both regions. Specifically, the average total transmit power decreased from $26 \mathrm{dBm}$ to 21 $\mathrm{dBm}\left(\sigma_{e}^{2}=0.01\right)$ at $R_{i}^{\mathrm{rsv}}=1$ in cell center region, and from $33 \mathrm{dBm}$ to $31 \mathrm{dBm}\left(\sigma_{e}^{2}=0.01\right)$ at $R_{i}^{\text {rvv }}=1$ in cell edge region, respectively. it also confirms that the greater value of the variance of SIC error is, the larger value of average total transmit power will be.

Figure 4 plots the average total transmit power versus probability of outage constraint, (i.e., $\epsilon$ ) for $R_{i}^{\text {rsv }}=0.1$ nats $/ \mathrm{s} / \mathrm{Hz}$ and $R_{i}^{\mathrm{rsv}}=1$ nats $/ \mathrm{s} / \mathrm{Hz}$. It can be seen clearly that the average total transmit power increases with increasing SIC error variance and rate reservation but decreases with less strict outage constraints. Furthermore, the total power consumption is less sensitive to different levels of SIC error variance at higher minimum reserved rate $R_{i}^{\text {rsv }}$.

Figure 5 demonstrates the experienced user outage versus probability of outage constraint, $\epsilon$. As expected, the experi-

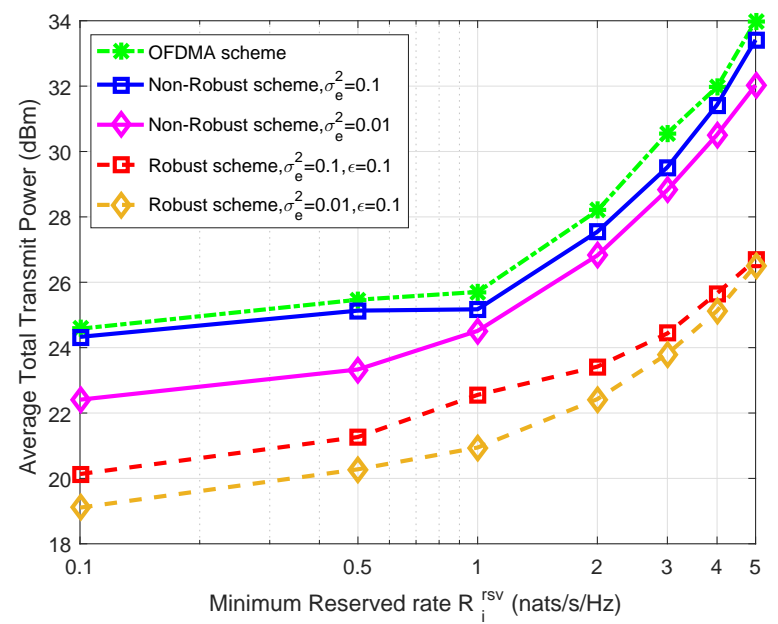

(a) Users at the cell center

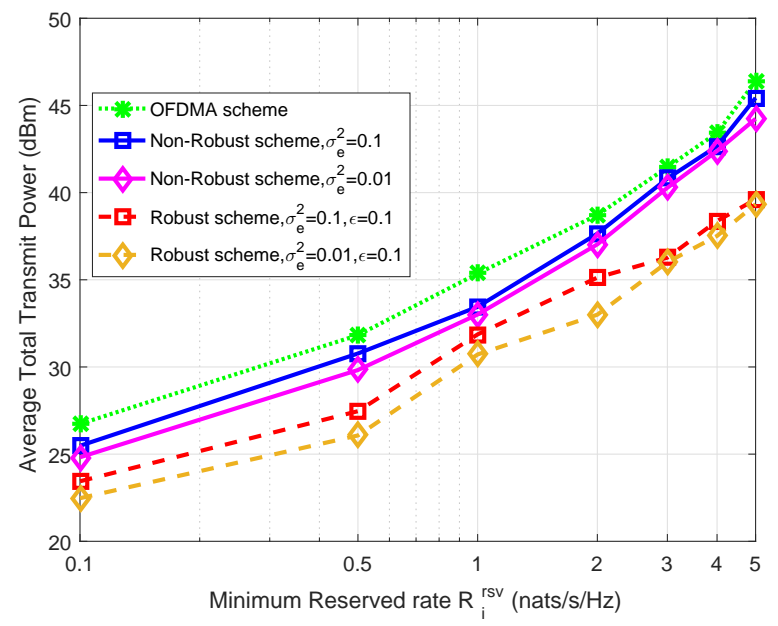

(b) Users at the cell edge

Fig. 3. Average total transmit power versus $R_{i}^{\text {rsv }}$

enced user outage increases proportional to outage constraints. We evaluate SIC imperfection for several levels of SIC error variance with $\sigma_{e}^{2}=0.01, \sigma_{e}^{2}=0.05$, and $\sigma_{e}^{2}=0.1$ and with different minimum reserved rate $R_{i}^{r s v}=0.1$ and 1 . It turns out that as the outage constraint is increased, the experienced user outage probability will be smoothly increased. For $R_{i}^{r s v}=0.1$, the experienced outage probability is much smaller than the probability used to constrain the problem $(\epsilon)$. For higher levels of minimum reserved rate (e.g., $R_{i}^{r s v}=1$ ), the feasibility of solutions can be impacted over the trial conducted and the resulting outage can differ from the constraint due to decreased feasibility of solutions.

\section{CONCLUSIONS}

In this paper, we have studied robust optimization for a downlink MC-NOMA network system under uncertainty introduced by SIC errors. The goal is to minimize the total transmit power subject to the outage probability of minimum 


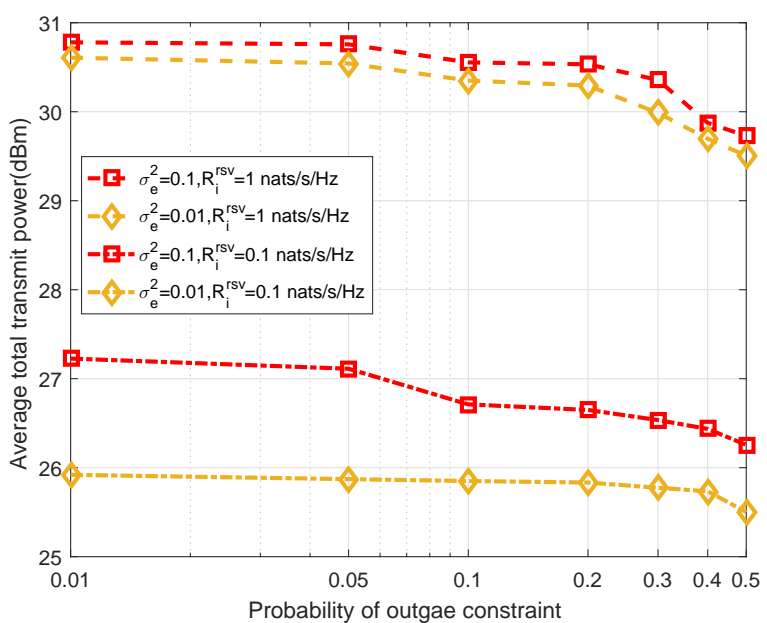

Fig. 4. Average total transmit power versus $\epsilon$

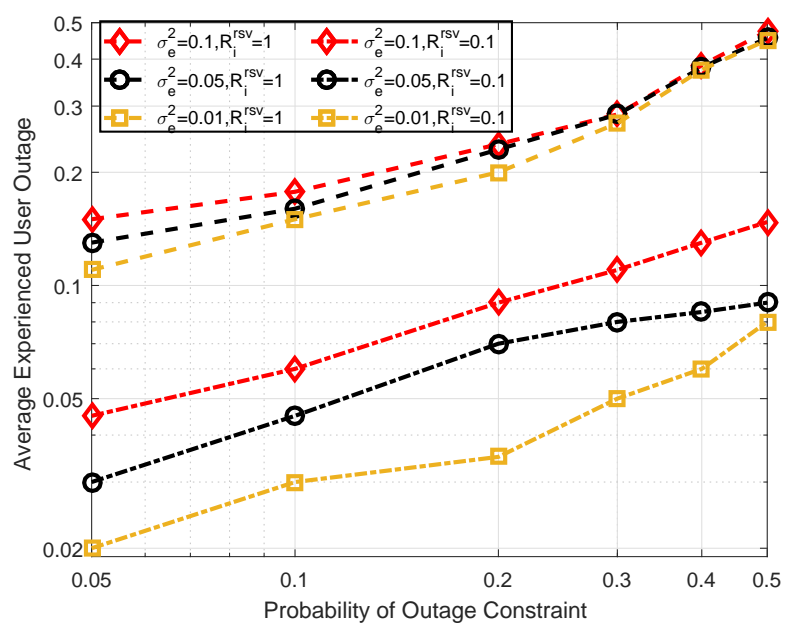

Fig. 5. Average experienced user outage versus $\epsilon$

reserved rate. In order to solve this non-convex problem, we have introduced the CGP and AGMA approximation methods to convert the original problem into a convex form. Then, we have investigated the impact of power allocation against: the number of users, minimum reserved rate and outage constraint. Next we have also studied the behavior of experienced user outage versus the outage constraint. Simulation results confirmed excellent performance of robust MC-NOMA system, in comparison to OFDMA system. Furthermore, we demonstrated that the proposed downlink robust MC-NOMA system provides significant power saving as compared to the non-robust scheme with the imperfect SIC.

\section{REFERENCES}

[1] L. Dai, B. Wang, Y. Yuan, S. Han, C. 1. I, and Z. Wang, "Non-orthogonal multiple access for 5G: solutions, challenges, opportunities, and future research trends," IEEE Communications Magazine, vol. 53, no. 9, pp. 74-81, Sep. 2015

[2] Y. Saito, A. Benjebbour, Y. Kishiyama, and T. Nakamura, "System-level performance evaluation of downlink non-orthogonal multiple access (NOMA)," in IEEE International Symposium on Personal, Indoor, and Mobile Radio Communications (PIMRC), Sep. 2013, pp. 611-615.

[3] Y. Saito, Y. Kishiyama, A. Benjebbour, T. Nakamura, A. Li, and K. Higuchi, "Non-orthogonal multiple access (NOMA) for cellular future radio access," in IEEE Vehicular Technology Conference (VTC Spring), Jun. 2013, pp. 1-5.

[4] S. Sen, N. Santhapuri, R. R. Choudhury, and S. Nelakuditi, "Successive interference cancellation: A back-of-the-envelope perspective," in $A C M$ SIGCOMM Workshop on Hot Topics in Networks, 2010, pp. 171-176.

[5] A. Benjebbour, Y. Saito, Y. Kishiyama, A. Li, A. Harada, and T. Nakamura, "Concept and practical considerations of non-orthogonal multiple access (NOMA) for future radio access," in International Symposium on Intelligent Signal Processing and Communication Systems, Nov. 2013, pp. $770-774$.

[6] Z. Ding, Y. Liu, J. Choi, Q. Sun, M. Elkashlan, C. L. I, and H. V. Poor, "Application of non-orthogonal multiple access in LTE and 5G networks," IEEE Communications Magazine, vol. 55, no. 2, pp. 185191, Feb. 2017.

[7] Z. Ding, P. Fan, and H. V. Poor, "Impact of user pairing on 5G nonorthogonal multiple-access downlink transmissions," IEEE Transactions on Vehicular Technology, vol. 65, no. 8, pp. 6010-6023, Aug. 2016.

[8] M. S. Ali, H. Tabassum, and E. Hossain, "Dynamic user clustering and power allocation for uplink and downlink non-orthogonal multiple access (NOMA) systems," IEEE Access, vol. 4, pp. 6325-6343, Aug. 2016.

[9] P. Parida and S. S. Das, "Power allocation in OFDM based NOMA systems: A DC programming approach," in IEEE Globecom Workshops (GC Wkshps), Dec. 2014, pp. 1026-1031.

[10] Z. Ding, R. Schober, and H. V. Poor, "On the design of MIMO-NOMA downlink and uplink transmission," in IEEE International Conference on Communications (ICC), May 2016, pp. 1-6.

[11] R. Dawadi, S. Parsaeefard, M. Derakhshani, and T. Le-Ngoc, "Powerefficient resource allocation in NOMA virtualized wireless networks," in IEEE Global Communications Conference (GLOBECOM), Dec. 2016, pp. 1-6.

[12] H. Sun, B. Xie, R. Q. Hu, and G. Wu, "Non-orthogonal multiple access with SIC error propagation in downlink wireless MIMO networks," in IEEE Vehicular Technology Conference (VTC-Fall), Sep. 2016, pp. 1-5.

[13] J. G. Andrews and T. H. Y. Meng, "Performance of multicarrier CDMA with successive interference cancellation in a multipath fading channel," IEEE Transactions on Communications, vol. 52, no. 5, pp. 811-822, May 2004.

[14] M. R. Usman, A. Khan, M. A. Usman, Y. S. Jang, and S. Y. Shin, "On the performance of perfect and imperfect SIC in downlink non orthogonal multiple access (NOMA)," in 2016 International Conference on Smart Green Technology in Electrical and Information Systems (ICSGTEIS), Oct 2016, pp. 102-106.

[15] M. Avriel and A. Williams, "Complementary geometric programming," SIAM Journal on Applied Mathematics, vol. 19, no. 1, pp. 125-141, 1970.

[16] G. Xu, "Global optimization of signomial geometric programming problems," European Journal of Operational Research, vol. 233, no. 3, pp. $500-510,2014$.

[17] M. Derakhshani, X. Wang, T. Le-Ngoc, and A. Leon-Garcia, "Airtime usage control in virtualized multi-cell 802.11 networks," in Globecom Workshops (GC Wkshps), 2015 IEEE. IEEE, 2015, pp. 1-6.

[18] H. Haci, H. Zhu, and J. Wang, "Performance of non-orthogonal multiple access with a novel asynchronous interference cancellation technique," IEEE Transactions on Communications, vol. 65, no. 3, pp. 1319-1335, March 2017.

[19] M. Chiang et al., "Geometric programming for communication systems," Foundations and Trends in Communications and Information Theory, vol. 2, no. 1-2, pp. 1-154, 2005.

[20] M. Grant, S. Boyd, and Y. Ye, "CVX: Matlab software for disciplined convex programming," 2008 